\title{
Embedded Green-function approach to the ballistic electron transport through an interface
}

\author{
D. Wortmann, ${ }^{1, *} \mathrm{H}$. Ishida, ${ }^{2}$ and S. Blügel ${ }^{3}$ \\ ${ }^{1}$ Institut für Festkörperforschung, Forschungszentrum Jülich, Jülich 52425, Germany \\ ${ }^{2}$ College of Humanities and Sciences, Nihon University, Sakura-josui, Tokyo 156, Japan \\ ${ }^{3}$ Fachbereich Physik, Universität Osnabrück, Osnabrück 49069, Germany
}

(Received 26 April 2002; published 15 August 2002)

\begin{abstract}
We present an efficient method for calculating the conductance of ballistic electrons through an interface from first principles using the embedding approach of Inglesfield. In our method the Landauer-Büttiker formula for ballistic transport is expressed in terms of two quantities that are available in the embedded Green-function formalism without additional calculations. One is the embedding potential of bulk crystals on both sides of the interface and the other is the Green function in the interface region. As a proof of principle we calculate on the basis of the density-functional theory the spin-resolved electron transmission through a model system of ferromagnetic $\mathrm{Co}$ monolayers sandwiched between bulk $\mathrm{Cu}$ crystals. The relationship between our formulation and the Green-function formulation of Baranger and Stone is discussed.
\end{abstract}

DOI: 10.1103/PhysRevB.66.075113

PACS number(s): 71.15.Ap, 73.40.-c, 72.10.-d, 73.20.At

\section{INTRODUCTION}

Studying electron transport through a thin interface layer or through a nanoscale contact between two electrodes forms a base of future technologies such as molecular electronics and spin electronics. According to the Landauer-Büttiker formula for ballistic transport, ${ }^{1}$ the conductance across the interface is determined by the transmission probability of electrons at the Fermi energy $\epsilon_{F}$. In other words, the conductance calculation is reduced to a scattering problem for an electron impinging on the interface. Another interesting subject related to such a calculation is the interaction between these interfaces. A recent example is the magnetic interlayer coupling, ${ }^{2,3}$ where the exchange-coupling strength between two magnetic films separated by a thin spacer layer can be estimated from the reflectivity of electrons at the interface between magnetic and spacer layers. ${ }^{4-6}$

In the past several electronic-structure methods were developed to address the problem of electron transmission and reflection at the interface. Among them are the tight-binding formulations, ${ }^{7-11}$ the Korringa-Kohn-Rostoker (KKR) Green-function approaches, ${ }^{12-14}$ and also the methods in which the Schrödinger equation was integrated on real-space mesh points across the interface. ${ }^{15-17}$ Based on a Greenfunction formulation, we recently developed a first-principles method to calculate the transfer matrix, ${ }^{18}$ which relates the value and normal derivative of an electron wave function on a boundary surface of a slab-shaped region to the corresponding ones on the opposite surface of the slab. As a first application we calculated complex band structures of bulk crystals. The method is also applicable to the calculation of electron transmission through an interface. This approach of matching wave functions seems straightforward requires, however, in practice quite some effort to reach numerical stability. ${ }^{19,20}$

The embedding approach of Inglesfield ${ }^{21}$ also provides a general framework to treat electronic states at the interface sandwiched between two bulk systems. In this method one introduces a so-called embedding surface weaving between the interface and bulk regions. Inglesfield showed that the effects of the bulk crystals beyond the embedding surfaces on both sides of the interface can be represented by an energy-dependent potential acting on the embedding surfaces. By simply adding the matrix elements of the embedding potentials to the Hamiltonian of an isolated slab, one can obtain the Green function of the entire system. So far the embedding method was mainly used for the ground-state electronic-structure calculations of interfaces and surfaces. ${ }^{22-25}$ In the present work we will show that the method is also suitable for transport calculations. We reformulate the Landauer-Büttiker formula ${ }^{1}$ to derive a very convenient expression that contains only the embedding potentials of the bulk systems and the boundary values of the interface Green function. Both quantities are available in a standard embedded Green-function calculation, and thus, it is possible to evaluate the conductance for ballistic transport without any additional computation.

To demonstrate the present formulation, we calculate the spin-resolved electron transmission through ferromagnetic fcc Co layers sandwiched between two semi-infinite Cu crystals in the [001] orientation. This is one of the most frequently studied systems in connection with magnetic interlayer coupling. Based on a full-potential linearized augmented plane-wave (FLAPW) scheme, the present calculation may provide more reliable data than previous ones within the muffin-tin potential approximation, in particular, for open systems and low symmetries.

The plan of the paper is as follows. In Sec. II we reformulate the Landauer-Büttiker formula for ballistic transport in terms of the embedding formalism. Section III contains numerical results for the $\mathrm{Cu} / \mathrm{Co} / \mathrm{Cu}$ sandwich system. Finally, a summary is given in Sec. IV. We use the Hartree atomic units throughout the paper unless otherwise stated.

\section{THEORY}

We consider a two-dimensional interface sandwiched between two bulk systems (see Fig. 1). $S_{\alpha}$ denotes a boundary surface between the interface region $\Omega$ and the bulk crystal $V_{\alpha}(\alpha=1,2)$. From the embedding point of view, $S_{\alpha}$ may be 


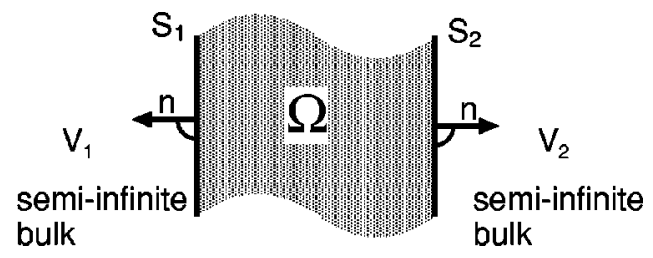

FIG. 1. Setup used in embedding calculations. The region of interest is a slab $\Omega$ between the two boundaries $S_{1}$ and $S_{2} . \Omega$ is infinite in two dimensions and of finite size normal to the boundaries. Outside the region $\Omega$ two semi-infinite bulk regions $V_{1}$ and $V_{2}$ are attached.

regarded as an embedding surface. We consider the oneelectron Green function with a real energy $\epsilon$,

$$
\left[-\frac{1}{2} \Delta+v(\vec{r})-\epsilon\right] G\left(\vec{r}, \vec{r}^{\prime} ; \epsilon\right)=-\delta\left(\vec{r}-\vec{r}^{\prime}\right),
$$

where $v(\vec{r})$ denotes the one-electron potential. For the time being, we do not specify the boundary condition of the Green function. Applying Green's theorem, one may show that $\psi(\vec{r})$, a solution of the Schrödinger equation,

$$
\left[-\frac{1}{2} \Delta+v(\vec{r})-\epsilon\right] \psi(\vec{r})=0
$$

for energy $\epsilon$ satisfies

$$
\psi(\vec{r})=-\frac{1}{2} \sum_{\alpha=1,2} \int_{S_{\alpha}}\left[G(\vec{r}, \vec{x}) \partial_{n} \psi(\vec{x})-\partial_{n} G(\vec{r}, \vec{x}) \psi(\vec{x})\right] d \vec{x}
$$

where $\vec{r}$ is any position in region $\Omega, \vec{x}$ is any position at the interface $S_{\alpha}$, and $\partial_{n}$ denotes a surface normal derivative with $n$ pointing outwards from $\Omega$. In Eq. (3) we omitted the energy dependence of the Green function for simplicity.

In bulk crystals, $V_{1}$ and $V_{2}$, the solutions of the Schrödinger equation are classified into those satisfying the incoming boundary condition and those satisfying the outgoing boundary condition. The solutions in each group are further divided into propagating Bloch states and evanescent waves. In the following we use the first superscript, 1 or 2 , to distinguish between states in $V_{1}$ and those in $V_{2}$, the second superscript, $i$ or $o$, to distinguish between incoming and outgoing states, and the subscript, $b$ or $e$, to distinguish between Bloch and evanescent waves. For example, $\psi_{b}^{1 i}(\vec{r})$ stands for a Bloch state in volume $V_{1}$ propagating toward the interface. Let us define an expectation value of the normal component of the current operator on $S_{\alpha}$ by

$$
J_{\alpha}(\phi, \psi)=\frac{1}{2 i} \int_{S_{\alpha}}\left[\phi^{*}(\vec{x}) \partial_{n} \psi(\vec{x})-\partial_{n} \phi^{*}(\vec{x}) \psi(\vec{x})\right] d \vec{x}
$$

$J_{\alpha}(\phi, \psi)$ remains constant if $S_{\alpha}$ is moved around inside the bulk crystal suppose that $\phi$ and $\psi$ are solutions of Eq. (2). This property leads to well-known identities,

$$
J_{\alpha}\left(\psi_{e}^{\alpha o}, \psi_{e^{\prime}}^{\alpha o}\right)=0
$$

$$
\begin{gathered}
J_{\alpha}\left(\psi_{e}^{\alpha o}, \psi_{b}^{\alpha o}\right)=J_{\alpha}\left(\psi_{b}^{\alpha o}, \psi_{e}^{\alpha o}\right)=0, \\
J_{\alpha}\left(\psi_{b}^{\alpha o}, \psi_{b^{\prime}}^{\alpha o}\right)=\delta_{b b^{\prime}}
\end{gathered}
$$

where Eq. (5c) provides a condition for normalizing the Bloch states.

Here it may be appropriate to introduce the "embedding potential" of Inglesfield, ${ }^{21}$ which relates the value and normal derivative on $S_{\alpha}$ of an outgoing solution of the Schrödinger equation with energy $\epsilon$ by

$$
\partial_{n} \psi_{\nu}^{\alpha o}(\vec{x})=2 \int_{S_{\alpha}} G_{S_{\alpha}}^{-1}\left(\vec{x}, \vec{x}^{\prime}\right) \psi_{\nu}^{\alpha o}\left(\vec{x}^{\prime}\right) d \vec{x}^{\prime},
$$

where $\nu$ represents either a Bloch or an evanescent state and we adopt a conventional notation $G_{S_{\alpha}}^{-1}$ for the embedding potential. Originally, the embedding potential was introduced by Inglesfield as a surface inverse of the Green function, which is defined in $V_{\alpha}$, fulfills the outgoing boundary condition in infinity, and has a vanishing normal derivative on $S_{\alpha}$. By substituting Eq. (6) in Eq. (4) and making use of the identity $G_{S_{\alpha}}^{-1}\left(\vec{x}, \vec{x}^{\prime}\right)=G_{S_{\alpha}}^{-1}\left(\vec{x}^{\prime}, \vec{x}\right)$, we obtain a useful expression,

$J_{\alpha}\left(\psi_{\nu}^{\alpha o}, \psi_{\nu^{\prime}}^{\alpha o}\right)=2 \int_{S_{\alpha}}\left[\psi_{\nu}^{\alpha o}(\vec{x})\right]^{*} \Im G_{S_{\alpha}}^{-1}\left(\vec{x}, \vec{x}^{\prime}\right) \psi_{\nu^{\prime}}^{\alpha o}\left(\vec{x}^{\prime}\right) d \vec{x} d \vec{x}^{\prime}$

Similarly, one may define a Wronskian-like quantity $W_{\alpha}(\phi, \psi)$ by replacing $\phi^{*}(\vec{r})$ in Eq. (4) by $\phi(\vec{r})$. In this case, instead of Eq. (7), one has $W_{\alpha}\left(\psi_{\nu}^{\alpha o}, \psi_{\nu^{\prime}}^{\alpha o}\right)=0$.

Now we consider a scattering process in which a Bloch state $b$ incident from the interior of the bulk crystal $V_{1}$ is scattered at the interface and either reflected back into $V_{1}$ or transmitted into $V_{2}$. The corresponding wave function can be expressed as

$$
\psi(\vec{r})= \begin{cases}\psi_{b}^{1 i}(\vec{r})+\sum_{\nu} r_{b \nu} \psi_{\nu}^{1 o}(\vec{r}), & \vec{r} \text { in } V_{1} \\ \sum_{\nu^{\prime}} t_{b \nu^{\prime}} \psi_{\nu^{\prime}}^{2 o}(\vec{r}), & \vec{r} \text { in } V_{2},\end{cases}
$$

where $r_{b \nu}$ and $t_{b \nu^{\prime}}$ denote reflection and transmission coefficients, respectively, with subscripts $\nu$ and $\nu^{\prime}$ running through both Bloch and evanescent states. We now specify the boundary condition of the Green function and assume that $G(\vec{r}, \vec{x})$ in Eq. (3) fulfills the outgoing boundary condition on $S_{1}$ and $S_{2}$. In this case the reflected and transmitted waves in Eq. (8) do not contribute to the integral in Eq. (3) because $W\left(\psi_{\nu}^{\alpha o}, \psi_{\nu^{\prime}}^{\alpha o}\right)=0$. As a result, Eq. (3) is simplified to

$$
\psi(\vec{r})=-\frac{1}{2} \int_{S_{1}}\left[G(\vec{r}, \vec{x}) \partial_{n} \psi_{b}^{1 i}(\vec{x})-\partial_{n} G(\vec{r}, \vec{x}) \psi_{b}^{1 i}(\vec{x})\right] d \vec{x},
$$

In deriving Eq. (9), it was assumed that $\vec{r}$ is located in $\Omega$. Yet, since $S_{2}$ can be moved to any place in the bulk crystal $V_{2}$, Eq. (9) holds for an arbitrary $\vec{r}$ in $V_{2}$. Also, $S_{1}$ can be 
moved to any place in $V_{1}$. The Green function with the outgoing boundary condition can be expanded as

$$
G\left(\vec{r}, \vec{r}^{\prime}\right)=\sum_{\nu, \nu^{\prime}} g_{\nu \nu^{\prime}} \psi_{\nu}^{1 o}(\vec{r}) \psi_{\nu^{\prime}}^{2 o}\left(\vec{r}^{\prime}\right),
$$

for $\vec{r}$ in $V_{1}$ and $\vec{r}^{\prime}$ in $V_{2}$. Let us denote the complex conjugate of $\psi_{b}^{1 i}(\vec{r})$ by $\psi_{b *}^{1 o}(\vec{r})$, which is a Bloch state propagating toward infinity. With this in mind, substituting Eq. (10) in Eq. (9) and using the identity $G\left(\vec{r}, \vec{r}^{\prime}\right)=G\left(\vec{r}^{\prime}, \vec{r}\right)$, one has

$$
\psi(\vec{r})=\sum_{\nu^{\prime}} i g_{b^{*} \nu^{\prime}} \psi_{\nu^{\prime}}^{2 o}(\vec{r}), \quad \vec{r} \text { in } V_{2} .
$$

Comparing Eq. (11) and Eq. (8) yields

$$
t_{b \nu^{\prime}}=i g_{b * \nu^{\prime}} .
$$

According to the formulation of Landauer and Büttiker, ${ }^{1}$ the conductance $\Gamma$ of ballistic electrons tunneling at a small bias voltage through the interface $\Omega$ can be expressed in terms of the transmission probability of all the Bloch states at the Fermi energy $\epsilon_{F}$ as

$$
\Gamma=\frac{1}{2 \pi} \sum_{b b^{\prime}}\left|t_{b b^{\prime}}\right|^{2}=\frac{1}{2 \pi} \sum_{b^{*} b^{\prime}}\left|g_{b^{*} b^{\prime}}\right|^{2} .
$$

With the use of Eq. (5), the above equation may read

$$
\Gamma=\frac{1}{2 \pi} \sum_{\mu \nu} \sum_{\mu^{\prime} \nu^{\prime}} g_{\mu \nu}^{*} J_{1}\left(\psi_{\mu}^{1 o}, \psi_{\mu^{\prime}}^{1 o}\right) J_{2}\left(\psi_{\nu}^{2 o}, \psi_{\nu^{\prime}}^{2 o}\right) g_{\mu^{\prime} \nu^{\prime}} .
$$

Using Eqs. (7) and (10), one obtains

$$
\begin{aligned}
\Gamma= & \frac{2}{\pi} \int_{S_{1}} d \vec{x}_{1} d \vec{x}_{1}^{\prime} \int_{S_{2}} d \vec{x}_{2} d \vec{x}_{2}^{\prime} G(1,2) \\
& \times \Im G_{S_{2}}^{-1}\left(2,2^{\prime}\right) G^{*}\left(2^{\prime}, 1^{\prime}\right) \Im G_{S_{1}}^{-1}\left(1^{\prime}, 1\right),
\end{aligned}
$$

where argument 1 in the Green function stands for $\vec{x}_{1}$ and the same applies for the other three arguments. With an abbreviation $G_{12}=G\left(\vec{x}_{1}, \vec{x}_{2}\right)$, Eq. (15) is written more concisely as

$$
\Gamma=\frac{2}{\pi} \operatorname{Tr}\left[G_{12} \Im G_{S_{2}}^{-1} G_{21}^{*} \Im G_{S_{1}}^{-1}\right],
$$

which is a main result of the present paper. In the embedding theory, the Green function satisfying the outgoing boundary condition is calculated by adding the matrix elements of the embedding potentials, $G_{S_{1}}^{-1}$ and $G_{S_{2}}^{-1}$, to the Hamiltonian of the isolated slab region $\Omega$. The imaginary part of such a Green function is related to the density of states of the system by

$$
n(\vec{r} ; \epsilon)=-\frac{2}{\pi} \Im G(\vec{r}, \vec{r} ; \epsilon) .
$$

Equation (16) adds a new field of application to the embedding method of Inglesfield beyond self-consistent electronicstructure calculations of surfaces and interfaces. It should be emphasized that all the quantities appearing in Eq. (16) are present in the embedded Green-function approach without performing any additional computation. One only has to calculate the Green function at $\epsilon_{F}$ to evaluate the conductance $\Gamma$ after self-consistency in the charge density is reached. On the other hand, it may be worth noting that with the embedding formalism alone, one cannot treat systems with a large bias voltage $\Delta V$ applied between the two bulk crystals on both sides of the interface. To be concrete, consider a case where $\epsilon_{F 1}=\epsilon_{F 2}+\Delta V$ with $\epsilon_{F \alpha}$ being the Fermi energy of bulk crystal $V_{\alpha}$ and $\Delta V>0$. Then, in the energy interval $\left[\epsilon_{F 2}, \epsilon_{F 1}\right]$, only the states incident from the interior of the bulk crystal $V_{1}$ contribute to the charge and current densities of the system, whereas the density of states as obtained from Eq. (17) contains also the contribution of the states incident from the interior of $V_{2}$. Hence, one must treat one-electron wave functions explicitly. One possibility for doing so is Eq. (9). Since the asymptotic form of the incident wave function, $\psi_{b}^{1 i}$, is known, one may evaluate $\psi(\vec{r})$ in the interface $\Omega$ from Eq. (9).

In the past, a number of papers have been published in which the the Landauer-Büttiker formula Eq. (13) was reformulated in terms of the Green function. Among them Baranger and Stone derived a general conductance formula ${ }^{26}$ that can be applied to multiple leads in a strong magnetic field. For the present system having two leads on both sides of the interface and without a magnetic field, their formula reads

$$
\begin{aligned}
\Gamma= & -\frac{1}{4 \pi} \int_{S_{1}} d \vec{x}_{1} \int_{S_{2}} d \vec{x}_{2} \Re\left[\partial_{1} \partial_{2} G(1,2)\right. \\
& \left.\times G^{*}(1,2)-\partial_{1} G(1,2) \partial_{2} G^{*}(1,2)\right],
\end{aligned}
$$

where $\partial_{\alpha}$ denotes an abbreviation of $\partial_{n}$ on $S_{\alpha}$ and it is understood that the Green function satisfies the outgoing boundary condition. To proceed, we express the normal derivatives of the Green function using the embedding potentials as

$$
\begin{aligned}
\partial_{1} G(1,2)= & 2 \int_{S_{1}} d \vec{x}_{1}^{\prime} G_{S_{1}}^{-1}\left(1,1^{\prime}\right) G\left(1^{\prime}, 2\right), \\
\partial_{2} G(1,2)= & 2 \int_{S_{2}} d \vec{x}_{2}^{\prime} G_{S_{2}}^{-1}\left(2,2^{\prime}\right) G\left(1,2^{\prime}\right), \\
\partial_{1} \partial_{2} G(1,2)= & 4 \int_{S_{1}} d \vec{x}_{1}^{\prime} \int_{S_{2}} d \vec{x}_{2}^{\prime} \\
& \times G_{S_{1}}^{-1}\left(1,1^{\prime}\right) G_{S_{2}}^{-1}\left(2,2^{\prime}\right) G\left(1^{\prime}, 2^{\prime}\right) .
\end{aligned}
$$

By substituting Eq. (19) in Eq. (18) and noting that the arguments in the Green functions and the embedding potentials are permutable, one can show easily that Eq. (18) coincides with Eq. (16). Baranger and Stone themselves gave a proof that Eq. (18) is equivalent to the Landauer-Büttiker formula. Their derivation is similar but different from ours leading to Eq. (16) in two points. First, they considered only propagating Bloch states in asymptotic regions, whereas we also took 
account of the evanescent waves in bulk regions. Second, we derived Eq. (9) using a Green's theorem, while they used another equation that holds for a wave function of a scattered state.

Two more comments are in order: (i) The formulas Eqs. (16) and (18) require the evaluation of the Green functions on boundary surfaces, $S_{1}$ and $S_{2}$. It was previously shown for a one-dimensional model ${ }^{27}$ that the Green function calculated with the embedding method is more accurate on boundary surfaces than that obtained by solving the Dyson equation cast in matrix form using basis functions, since the embedding method explicitly takes account of the outgoing boundary condition of the Green function. (ii) Even with the embedding method, it would be extremely difficult to numerically compute the derivatives of the Green function on boundary surfaces, since typically, the Green function is expanded with a finite number of plane-wave basislike functions in actual implementations of the embedding approach. In such an expansion, the numerical derivative converges much slower that the function itself and oscillations in the current density might occur, which violate current conservation. With Eq. (16) one can avoid these problems since no numerical derivative of the Green function has to be calculated. The embedding potential in Eq. (16) is a property of the bulk crystal and should be calculated separately prior to the embedding calculation. We have recently developed a very accurate method to calculate the embedding potential from the complex electronic band structure of a bulk crystal. $^{18}$

Before closing this section, we would like to stress an interesting aspect of our formalism. Even though we referred to the volumes $V_{1}$ and $V_{2}$ attached to region $\Omega$ as semiinfinite bulk regions so far, this is not a necessary condition, in fact the potential in these regions only has to be that of a periodic bulk crystal far away from the embedding surfaces. If the potential differs from the bulk potential near the embedding surface, Eq. (16) can still be applied. The only change in the derivation of the equation would be the naming of the states. One would no longer speak of Bloch and evanescent waves of the bulk but instead of states which carry a current (do not carry a current) and are obtained from Bloch states (evanescent states) by integrating those from the bulk towards the embedding plane, respectively. Thus, by shrinking region $\Omega$ and with new boundaries, Eq. (16) can still be applied on these boundaries by transferring the embedding potentials to the new embedding surfaces using the embedding method itself. ${ }^{22,24}$ A particularly simplified limit of this procedure is reached, when $S_{1}$ and $S_{2}$ coincide with each other.

For simplicity, we consider $S_{1}$ and $S_{2}$ to be planar interfaces with the $z$ axis pointing normal to both the planes. $S_{1}$ and $S_{2}$ are chosen as $z=z_{1}$ and $z=z_{2}$, respectively, where we take the limit of $z_{2}-z_{1} \rightarrow 0$ in the very end. Integrating Eq. (1) along the surface normal direction leads to the cusp condition,

$$
\left[\lim _{z \rightarrow z^{\prime}+0}-\lim _{z \rightarrow z^{\prime}-0}\right] \partial_{z} G\left(\vec{x}, z, \vec{x}^{\prime}, z^{\prime}\right)=2 \delta\left(\vec{x}-\vec{x}^{\prime}\right),
$$

where $z_{1}<z^{\prime}<z_{2}$ and $\vec{x}=(x, y)$ are the planar coordinates. In the limit of $z_{2}-z_{1} \rightarrow 0$, the first term of the left-hand side of Eq. (20) may be replaced by $\partial_{z_{2}} G\left(\vec{x}, z_{2}, \vec{x}^{\prime}, z_{1}\right)$, while the second term by $\partial_{z_{1}} G\left(\vec{x}, z_{1}, \vec{x}^{\prime}, z_{2}\right)$. Then, expressing the normal derivatives of the Green function in terms of the embedding potentials yields

$$
\begin{aligned}
& \int_{S_{2}} d \vec{x}^{\prime \prime} G_{S_{2}}^{-1}\left(\vec{x}, \vec{x}^{\prime \prime}\right) G\left(\vec{x}^{\prime \prime}, z_{2}, \vec{x}^{\prime}, z_{1}\right) \\
& \quad+\int_{S_{1}} d \vec{x}^{\prime \prime} G_{S_{1}}^{-1}\left(\vec{x}, \vec{x}^{\prime \prime}\right) G\left(\vec{x}^{\prime \prime}, z_{1}, \vec{x}^{\prime}, z_{2}\right)=\delta\left(\vec{x}-\vec{x}^{\prime}\right)
\end{aligned}
$$

Furthermore, in the limit of $z_{2}-z_{1} \rightarrow 0, G\left(\vec{x}^{\prime \prime}, z_{2}, \vec{x}^{\prime}, z_{1}\right)$ in the first term of the left-hand side of Eq. (21) becomes identical with $G\left(\vec{x}^{\prime \prime}, z_{1}, \vec{x}^{\prime}, z_{2}\right)$. Thus, Eq. (21) reads in matrix form as

$$
\left[G_{S_{1}}^{-1}+G_{S_{2}}^{-1}\right] G_{12}=1 .
$$

By combining Eqs. (16) and (22), we now obtain

$$
\Gamma=\frac{2}{\pi} \operatorname{Tr}\left\{\left[G_{S_{1}}^{-1}+G_{S_{2}}^{-1}\right]^{-1} \Im G_{S_{2}}^{-1}\left[G_{S_{1}}^{-1}+G_{S_{2}}^{-1}\right]^{*-1} \Im G_{S_{1}}^{-1}\right\} .
$$

As expected, the conductance for ballistic transport in this limit can be expressed only in terms of the embedding potentials of the two bulk crystals on both sides of the boundary surface. It may be obvious from the above derivation that Eq. (23) holds also for any curvy boundary surface. As the simplest example, let us consider a one-dimensional step potential where $v(z)=0$ in $V_{1}(z<0)$ and $v(z)=v_{0}$ in $V_{2}(z$ $>0$ ). First, we choose the boundary surface for the embedding potentials as $z=0$. Then, for an electron with energy $\epsilon$ $\left(>v_{0}>0\right), G_{S_{1}}^{-1}=i k_{1} / 2$ and $G_{S_{2}}^{-1}=i k_{2} / 2$ where $k_{1}=\sqrt{2 \epsilon}$ and $k_{2}=\sqrt{2\left(\epsilon-v_{0}\right)}$ Eq. (23) reproduces a result of elementary quantum mechanics for the transmission probability $\mathrm{T}$,

$$
T=2 \pi \Gamma=\frac{4 k_{1} k_{2}}{\left(k_{1}+k_{2}\right)^{2}} .
$$

As discussed above, the boundary surface between the two half-spaces do not need to be located at the boundary of the two bulk regions. If it is shifted to $z=-d$ as measured from the potential step, $G_{S_{1}}^{-1}$ remains the same, whereas the embedding potential for the right half-space now becomes

$$
G_{S_{2}}^{-1}=\frac{i k_{1}}{2} \frac{k_{2} \cos \left(k_{1} d\right)-i k_{1} \sin \left(k_{1} d\right)}{k_{1} \cos \left(k_{1} d\right)-i k_{2} \sin \left(k_{1} d\right)} .
$$

We emphasize that in this case the space to the right of $S_{2}$ $\left(=S_{1}\right)$, i.e., $z>-d$ cannot be regarded as a bulk region any more because of the potential step at $z=0$. In spite of this, by inserting Eq. (25) in Eq. (23), one obtains for $\Gamma$ exactly the same expression (24). 


\section{RESULTS}

In this section we present a first application of Eq. (16) to a realistic system. To check the accuracy and to allow a comparison with previous calculations we have chosen the $\mathrm{Cu} / \mathrm{Co} / \mathrm{Cu}(001)$ sandwich structure that is a model system in magnetoelectronics and intensively investigated before. $5,13,28$

We implemented the embedding formalism within the framework of the FLAPW method. Like in standard FLAPW electronic-structure calculations we introduce a finite basis set of functions with a different representation within muffintin (MT) spheres around the atoms and the remaining interstitial region,

$$
\phi_{\vec{k}_{\|}, \vec{g}}(\vec{r})=\frac{1}{\sqrt{A}} \begin{cases}e^{i\left(\overrightarrow{k_{\|}}+\vec{g}_{\|}\right) \vec{x}+g_{z} z}, & \vec{r} \in \mathrm{Int} \\ \phi_{\vec{k}_{\|}, \vec{g}}^{M T}(\vec{r}), & \vec{r} \in \mathrm{MT},\end{cases}
$$

where $A$ denotes a normalization area. Our systems possess a two-dimensional (2D) translational symmetry and thus the problem can be solved independently for each $2 \mathrm{D}$ wave vector $\vec{k}_{\|}$from the 2D Brillouin zone. The 2D vectors $\vec{g}_{\|}$are reciprocal-lattice vectors according to the 2D translational symmetry of the problem and the third component $g_{z}$ $=2 \pi n / \widetilde{d}\left(n=-N_{\max }, \ldots, N_{\max }\right)$ in the direction perpendicular to the plane imposes a periodicity $\widetilde{d}$ slightly larger than the distance between $S_{1}$ and $S_{2}$. At this point an additional problem should be mentioned: While the LAPW-basis functions are known to be very well adopted to expand the Hamiltonian, the different representation of the basis functions introduces a significant problem in the case of closely spaced atomic layers as no planar embedding surface can be constructed without cutting muffin-tin spheres. The use of a curved surface or of an embedding surface cutting through muffin tins would make the actual implementation very cumbersome and prone to numerical inaccuracies. To overcome this limitation we introduce additional volumes to obtain planar embedding surfaces $S_{1}$ and $S_{2}$, as was proposed in Refs. $22,24,29$. More details on the resulting setup and the form of the basis functions can be found in Ref. 18. Using the additional volumes the embedding surfaces cut through the interstitial region only.

Using the LAPW-basis functions Eq. (1) turns into a matrix equation and the Green-function matrix is obtained by a matrix inversion. While this matrix is expanded into the full three-dimensional set of basis functions one should note that the $G_{12}$ as defined for Eq. (16) are given only on the 2D surfaces and thus can be expanded into a much smaller set of 2D plane waves with wave vectors $\vec{k}_{\|}+\vec{g}_{\|}$. Thus the application of Eq. (16) requires the multiplication of four 2D matrices where the embedding matrices are calculated separately.

The calculation was performed in three steps: (i) First we performed a supercell calculation containing 5 monolayers (ML) of $\mathrm{Cu}$ and $5 \mathrm{ML}$ of $\mathrm{Co}$ to obtain the spin-dependent potential used in the further steps. These were standard spinpolarized FLAPW calculations done with the FLEUR code. ${ }^{30}$ To enable a comparison of our results with those obtained from previous KKR-Green-function calculations ${ }^{13,28}$ we used

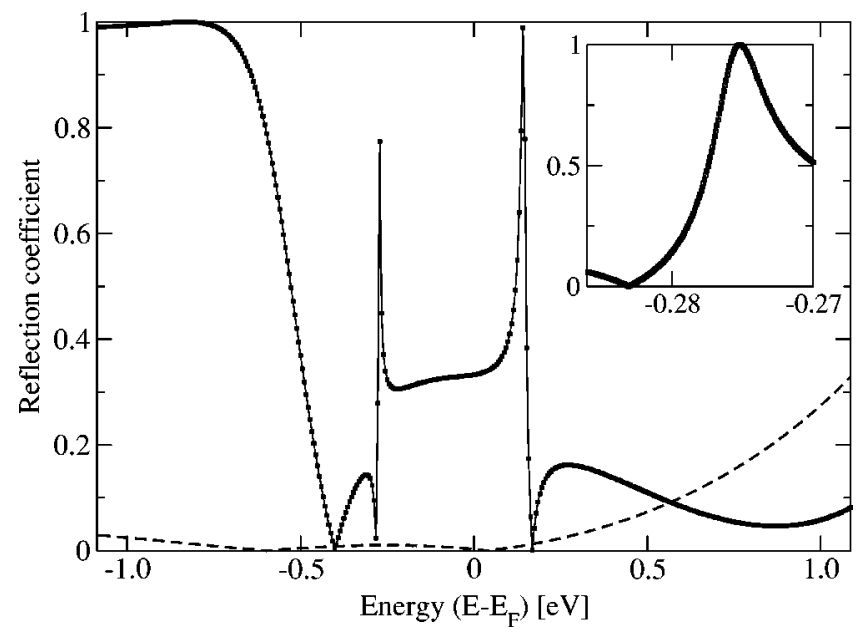

FIG. 2. Spin-resolved reflection coefficient for a ferromagnetic $\mathrm{Cu} / 5 \mathrm{ML} \mathrm{Co} / \mathrm{Cu}(001)$ layer for $\vec{k}_{\|}=0$. The zero of energy was chosen to be the Fermi level. Note that the resonance peaks actually reach a value of one as shown in the inset. The difference between two energy grid points was $5.5 \mathrm{meV}$ (inset: $0.08 \mathrm{meV}$ ).

the same lattice constant $(a=6.76$ a.u. $)$, the same atomic arrangement assuming a constant interlayer spacing ( $d$ $=3.38$ a.u.) throughout the system and also the local spindensity approximation to the exchange-correlation potential. $^{31}$ (ii) In a second step we used the potential of the central $\mathrm{Cu}$ layer to calculate the complex band structure and the embedding potential of bulk $\mathrm{Cu}$ using our $T$-matrix method. ${ }^{18}$ As the potential converges rapidly towards the bulk values, the results in this calculation are in good agreement to the results for semi-infinite bulk $\mathrm{Cu}$. (iii) Finally, we set up a slab of 9 ML thickness containing $5 \mathrm{ML}$ of $\mathrm{Co}$ in the center and two $\mathrm{Cu}$ layers on both sides. The embedding potential for bulk $\mathrm{Cu}$ was added on both sides to calculate eventually the spin-dependent Green function of $5 \mathrm{ML}$ of Co sandwiched between two semi-infinite $\mathrm{Cu}$ crystals. As the potential obtained from the bulk supercell calculation was used in this last calculation the potential exhibits no artificial discontinuities. The two $\mathrm{ML}$ of $\mathrm{Cu}$ included in this setup ensure a realistic treatment of the $\mathrm{Co}-\mathrm{Cu}$ interface as the potential varies smoothly over the interface. We checked the convergence of the result by varying the number of wave vectors perpendicular to the embedding plane from $\sim 70$ to $\sim 180$, leading to a variation in the total number of LAPWbasis function from $\sim 1400$ to $\sim 3800$, and found our results to be very well stable and well converged under those variations.

In general our formalism allows us to calculate the conductance $\Gamma\left(\vec{k}_{\|} ; \epsilon\right)$ for each energy $\epsilon$ and each vector $\vec{k}_{\|}$from the the 2D Brillouin zone for arbitrary numbers of incoming Bloch waves. However, if at most a single incoming Bloch state propagates toward the interface the reflection coefficient $\left|r\left(\vec{k}_{\|} ; \epsilon\right)\right|$ for this state and the conductance we obtain from Eq. (16) are related by

$$
\left|r\left(\vec{k}_{\|} ; \epsilon\right)\right|=\sqrt{1-2 \pi \Gamma\left(\vec{k}_{\|} ; \epsilon\right)} .
$$

Figure 2 depicts the spin-resolved reflection coefficients 
for $\mathrm{Cu}$ electrons scattered by $5 \mathrm{ML}$ of ferromagnetic Co determined by this equation. The dashed line corresponds to the majority spin channel while results for the minority spin are shown by the squares connected by solid lines. Only states with normal incidence $\left(\vec{k}_{\|}=0\right)$, i.e., states from the $\bar{\Gamma}$ point of the 2D Brillouin zone have been considered. In the majority spin channel the reflectivity of the Co layers is low and varies smoothly over a wide energy range. This is easily understood from the bulk band structures of $\mathrm{Cu}$ and $\mathrm{Co}^{28}$ which both show a single band of mostly $s$ character and the same 2D symmetry around the Fermi energy. For the minority spin, however, we find a strong energy dependence of the reflection coefficient. The nearly perfect reflectivity below $-0.7 \mathrm{eV}$ is due to the absence of Co states with the same 2D symmetry as the incoming $\mathrm{Cu}$ wave function. The most pronounced features in Fig. 2, however, are the sharp peaks at around $-0.28 \mathrm{eV}$ and $0.15 \mathrm{eV}$. These are due to Fano resonances in the Co layers. ${ }^{32} \mathrm{~A}$ more detailed discussion of Fig. 2 can, e.g., be found in the paper of Wildberger et al. ${ }^{13}$ Concerning the key issue of the calculation, the comparison between our results and previous ones, we can conclude that our results are in good overall agreement with those previously obtained ${ }^{13,28}$ for the same $\mathrm{Cu} / \mathrm{Co} / \mathrm{Cu}$ setup using the KKR method. A stringent test of the quality of our results is the verification of the reflectivity behavior at these resonances, which varies strongly from zero, i.e., total transmission, to exactly one, i.e., total reflection. The inset of Fig. 2, which shows only a small energy interval around the peak lower in energy but resolved with a high density of energy grid points, confirms that this behavior is reproduced very accurately in the present calculation. The deviation from total transmission or reflection at the peak positions is less than $10^{-2}$. This is different from the KKR results. They are obtained by carrying out calculations on the complex energy plane. This introduces a computational broadening of the resonances and simultaneously a reduction of the peak height from the ideal value. Instead, we work with real energies where the broadening of the peaks can be avoided.

\section{SUMMARY}

Based on the embedding approach of Inglesfield we developed an efficient method enabling us to treat ballistic transport of electrons on an $a b$ initio level. By rewriting the Landauer-Büttiker formula in terms of the Green function of the interface region and the embedding potentials we arrive at an equation that is easily applied within an embedding calculation. As a first example the formalism was applied to the transmission of $\mathrm{Cu}$ electrons through a thin Co layer, an example studied extensively before and a very good agreement with results of KKR calculations was shown. Further applications of the theory could include more complex systems in which also a self-consistent charge density calculated by the embedding method is used.

\section{ACKNOWLEDGMENTS}

D.W. thanks the DAAD and the HGF-Strategiefonds "Magnetoelectronics" for financial support.
*Electronic address: d.wortmann@fz-juelich.de

${ }^{1}$ M. Büttiker, Phys. Rev. Lett. 57, 1761 (1986).

${ }^{2}$ P. Grünberg, R. Schreiber, Y. Pang, M. Brodsky, and H. Sower, Phys. Rev. Lett. 57, 2442 (1986).

${ }^{3}$ S.S.P. Parkin, N. More, and K.P. Roche, Phys. Rev. Lett. 64, 2304 (1990).

${ }^{4}$ P. Bruno, Phys. Rev. B 52, 411 (1995).

${ }^{5}$ B. Lee and Y. Chang, Phys. Rev. B 52, 3499 (1995).

${ }^{6}$ M.D. Stiles, Phys. Rev. B 48, 7238 (1993).

${ }^{7}$ P. Sautet and C. Joachim, Phys. Rev. B 38, 12238 (1988).

${ }^{8}$ J. Ferrer, A. Martin-Rodero, and F. Flores, Phys. Rev. B 38, 10113 (1988).

${ }^{9}$ L. Chico, L.X. Benedict, S.G. Louie, and M.L. Cohen, Phys. Rev. B 54, 2600 (1996).

${ }^{10}$ J. Cerdá, M.A. Van Hove, P. Sautet, and M. Salmeron, Phys. Rev. B 56, 15885 (1997).

${ }^{11}$ J. Mathon, Phys. Rev. B 56, 11810 (1997).

${ }^{12}$ N. Kar and P. Soven, Solid State Commun. 19, 1041 (1976).

${ }^{13}$ K. Wildberger, R. Zeller, P.H. Dederichs, J. Kudrnovsky, and P. Weinberger, Phys. Rev. B 58, 13721 (1998).

${ }^{14}$ O. Wunnicke, P. Mavropoulos, R. Zeller, P.H. Dederichs, and D. Grundler, Phys. Rev. B 65, 241306 (2002); P. Mavropoulos, O. Wunnicke, and P.H. Dederichs, Phys. Rev. B 66, 024416 (2002).
${ }^{15}$ J.A. Appelbaum and D.R. Hamann, Phys. Rev. B 6, 2166 (1972).

${ }^{16}$ K. Hirose and M. Tsukada, Phys. Rev. Lett. 73, 150 (1994).

${ }^{17}$ K. Hirose and M. Tsukada, Phys. Rev. B 51, 5278 (1995).

${ }^{18}$ D. Wortmann, H. Ishida, and S. Blügel, Phys. Rev. B 65, 165103 (2002).

${ }^{19}$ G. Wachutka, Phys. Rev. B 34, 8512 (1986).

${ }^{20}$ M.D. Stiles and D.R. Hamann, Phys. Rev. B 38, 2021 (1988).

${ }^{21}$ J.E. Inglesfield, J. Phys. C 14, 3795 (1981).

${ }^{22}$ S. Crampin, J.B.A.N. van Hoof, M. Nekovee, and J.E. Inglesfield, J. Phys.: Condens. Matter 4, 1475 (1992).

${ }^{23}$ S. Crampin, M. Nekovee, J.B.A.N. van Hoof, and J.E. Inglesfield, Surf. Sci. 287/88, 732 (1993).

${ }^{24}$ H. Ishida, Surf. Sci. 388, 71 (1997).

${ }^{25}$ K. Schep, J. van Hoof, P. Kelly, G. Bauer, and J. Inglesfield, Phys. Rev. B 56, 10805 (1997).

${ }^{26}$ H.U. Baranger and A.D. Stone, Phys. Rev. B 40, 8169 (1989).

${ }^{27}$ H. Ishida and M.I. Trioni, Phys. Rev. B 63, 155108 (2001).

${ }^{28}$ I. Riedel, P. Zahn, and I. Mertig, Phys. Rev. B 63, 195403 (2001).

${ }^{29}$ H. Ishida, Phys. Rev. B 63, 165409 (2001).

${ }^{30} \mathrm{http} / / /$ www.flapw.de

${ }^{31}$ S. Vosko, L. Wilk, and N. Nusair, Can. J. Phys. 58, 1200 (1980).

${ }^{32}$ U. Fano, Phys. Rev. 124, 1866 (1961). 